\title{
Impact of Exposure to War Stress on Exacerbations of Multiple Sclerosis
}

\author{
Daniel Golan, MD, ${ }^{1}$ Eli Somer, PhD, ${ }^{2}$ Sara Dishon, RN, MPA, ${ }^{1}$ Limor Cuzin-Disegni, MA, ${ }^{1}$ \\ and Ariel Miller, $\mathrm{MD}, \mathrm{PhD}^{1,3}$
}

\begin{abstract}
Objective: To assess the relation between stress caused by the perils of rocket attack on civilian centers in northern Israel during the 2006 war between Hezbollah and Israel and multiple sclerosis (MS) exacerbations.

Methods: Participants were 156 patients with relapsing-remitting MS. We compared the number of relapses during and after the war with similar time periods at the preceding year. Exposure to war events and resulting subjective stress were evaluated by means of structured interviews using questionnaires previously validated.

Results: During the 33 days of the war, there were 18 relapses among our patients, compared with 1 to 6 relapses in similar time periods over the 12 months before the war $(p<0.001-0.02)$. There was no increase in relapse rate during the 3 months that followed the war $(p=0.58)$. The percentage of patients reporting the experience of intense subjective stress during the hostilities was significantly greater among patients with wartime relapse compared with the rest of the patients (44 vs 20\%). The proportion of patients reporting high levels of distress associated with exposure to rocket attacks, displacement from home, and perceived life threat was greater in relapsing patients compared with those in remission ( 67 vs $42 \%, p=0.05 ; 33$ vs $11 \%$, $p=$ 0.02 ; and 33 vs $15 \%, p=0.08$, respectively).

Interpretation: Our data suggest that civilian exposure to war stress is associated with increased risk for MS relapse. These findings provide insight to stress-related risk factors associated with relapses of MS.
\end{abstract}

Ann Neurol 2008;64:143-148

The notion that psychological stress may be related to multiple sclerosis (MS) dates back to the time of Charcot, who suggested that the onset of MS is often preceded by grief or vexation, as well as by other social undesirable circumstances. ${ }^{1}$

Over time several studies designed to test the alleged relation among stressful life events, psychological distress, and MS exacerbations have been performed. In 1999, a review of the literature was published by a task force of the American Academy of Neurology. ${ }^{2}$ The authors conclude that, although the relation between antecedent psychological stress and MS exacerbation is possible, the studies have significant limitations leaving the association equivocal. Among the identified issues of concern were lack of a clear biological model, the absence of an agreed-on measure of stress, recall bias in retrospective interviews, and conflicting results in various studies.

Since this last mentioned review, several important studies have been published, further supporting the contribution of psychological stress to MS disease activity. $^{3-7}$ In a meta-analysis of 14 studies on the sub- ject, a significantly increased risk for exacerbation associated with stressful life events was established. ${ }^{6}$

The relation between war stress and MS exacerbations has been evaluated previously in a small study of 32 MS patients from Israel during the Persian Gulf War of 1991. ${ }^{8}$ The authors report a significantly lower attack rate in the 40 days of that war, as well as in the following 2 months, compared with similar prewar time periods. However, the small number of patients studied has been a significant shortcoming, limiting the conclusiveness of its findings.

The purpose of this study was to assess the relation between war-related stress of civilians during the July to August 2006 shelling on Israel and MS exacerbations. During the 33 days of hostilities, some 4,000 rockets rained on the civilian population of northern Israel. An average of 120 rockets and mortar bombs exploded in this region every day. In these attacks, 43 civilians were killed, more than 4,260 were injured, and 2,773 (65\% of those evacuated to hospitals) were treated for posttraumatic stress. The extensive civic damage and the constant mortal risk motivated
From the ${ }^{1}$ Multiple Sclerosis Center and Department of Neurology, Carmel Medical Center; ${ }^{2}$ Faculty of Social Welfare and Health Studies, University of Haifa; and ${ }^{3}$ Rappaport Faculty of Medicine and Research Institute, Technion-Israel Institute of Technology, Haifa, Israel.

Received Nov 30, 2007, and in revised form Mar 3, 2008. Accepted for publication Mar 21, 2008.
Published online June 20, 2008, in Wiley InterScience (www.interscience.wiley.com). DOI: 10.1002/ana.21409

Address correspondence to Dr Miller, Multiple Sclerosis and Brain Research Center, Department of Neurology, Carmel Medical Center, Haifa 34362, Israel. E-mail: millera@tx.technion.ac.il 
300,000 civilians to evacuate their homes, sending more than a million citizens to live in bomb shelters.

\section{Patients and Methods}

\section{Study Population and Procedure}

Participants were 156 consecutive relapsing-remitting MS patients who attended our MS clinic for a prescheduled follow-up examination during the 4th month after the end of the war. All these visits were scheduled before the war's onset as part of the MS clinic's routine clinical follow-up program. No "emergency" (none prescheduled) visits were included. The 156 patients included in the study were patients who actually attended the scheduled visit at the 4th month after the war, of 175 prescheduled visits. This constitutes $89 \%$ compliance and is within the range of 85 to $90 \%$, which is the compliance rate at our MS center. This indicates a low "no-show" rate, and that our study group is accurately representative of our MS clinic's patient population. All participants had been examined routinely at our clinic, in addition to assessments at disease exacerbations, during the 12 months before the war. They were all residents of northern Israel and met the revised "McDonald diagnostic criteria." ${ }^{10}$

To track down all confirmed and significant relapses that happened during wartime, and to avoid inclusion of "pseudorelapses," we interviewed all patients using a validated questionnaire for MS symptoms and disability. ${ }^{11}$ We included in the analysis only those relapses that met the following three criteria: (1) they were characterized by emergence of new symptoms or the worsening of existing ones for more than 24 hours in the absence of fever or infection ${ }^{12}$; (2) they were described in the interview as inflicting "considerable functional impairment"; and (3) they were also documented in the patients' medical record as exacerbations that indicated corticosteroid treatment. We compared the number of confirmed relapses during and after the war with similar periods at the preceding year.

The dates of exacerbations in the 12 months before the war were extracted from the medical records. All these relapses were confirmed by a neurologist and were treated by corticosteroids.

The Helsinki commission of Carmel Medical Center, Haifa, Israel, approved the study. All patients provided informed consent.

\section{Exposure and Stress-Related Measurement}

Patients were presented with a list of potential wartime stressors and were asked to indicate whether they had been exposed to each traumatic event during the war and, if so, how subjectively distressful was it on a four-point Likert scale. The inquired war stressors included exposure to air-raid sirens warning of incoming rockets, displacement from home, perceived life threat, hiding in bomb shelters, home damaged by a rocket hit, physical injury, financial loss because of disruption of business, forced separation from close relatives because of their military recruitment or displacement, and the death of a family member or a friend during a rocket attack.

For further assessment of subjective stress during the war, we used the abridged four-item version of the "perceived stress scale." 13 The average score of the four items is a num-

\begin{tabular}{|c|c|}
\hline \multicolumn{2}{|c|}{ Table. Patient Characteristics $(\mathrm{N}=156)$} \\
\hline Sex, F/M & $121(78 \%) / 35(22 \%)$ \\
\hline Mean age $\pm S D$ (range), yr & $44 \pm 12(17-71)$ \\
\hline $\begin{array}{l}\text { Mean duration of illness } \\
\pm \mathrm{SD} \text { (range), yr }\end{array}$ & $10 \pm 7(1-35)$ \\
\hline $\begin{array}{l}\text { Mean EDSS score } \pm \text { SD } \\
\text { (range) }\end{array}$ & $2.8 \pm 1.8(0-6.5)$ \\
\hline \multicolumn{2}{|c|}{ Relapses from June 2005 to June 2006, n (\%) } \\
\hline 0 & $128(82)$ \\
\hline 1 & $18(12)$ \\
\hline $2-4$ & $10(6)$ \\
\hline Disease-modifying drugs, $\mathrm{n}(\%)$ & $126(81)$ \\
\hline \multicolumn{2}{|c|}{ Stayed home during the war, $\mathrm{n}(\%)$} \\
\hline At least few days & $144(92)$ \\
\hline More than 1 week & $120(77)$ \\
\hline Throughout the war & $113(72)$ \\
\hline $\begin{array}{l}\mathrm{SD}=\text { standard deviation; EDSS }= \\
\text { Scale. }\end{array}$ & Expanded Disability Status \\
\hline
\end{tabular}

ber between 1 and 4 (higher score denotes high subjective stress). The perceived stress scale has been designed to measure the degree to which situations in one's life are experienced as uncontrollable, unpredictable, and overwhelming and consequently stressful. This scale has been shown to be reliable and was criterion validated, demonstrating strong relations with other measures of stress. ${ }^{13}$

To control for adverse life events unrelated to the war, we asked our patients to indicate whether they had experienced other specific life events during the current war or in the preceding year. We addressed events that had been previously shown to be related to MS exacerbations ${ }^{14}$ : death in the family, marital and job conflicts, financial difficulties, major illness of oneself (other than MS), or major illness of a close relative.

\section{Statistical Analysis}

Statistical analysis was done using SPSS software (SPSS, Chicago, IL). Summary statistics are given as mean \pm standard deviation. We used the McNemar test to compare the number of confirmed relapses between the different months. $\chi^{2}$ and Fisher's exact tests were used for comparing the proportion of patients who mentioned high subjective wartime stress between the relapse and remission groups.

\section{Results}

\section{Patient Characteristics}

The study population consisted of 156 relapsingremitting MS patients (Table). There were 121 (78\%) women and $35(22 \%)$ men. The average age was $44 \pm$ 12 years old. Time since diagnosis was $10 \pm 7$ years with mean Expanded Disability Status Scale score of $2.8 \pm 1.8$. The majority of our population $(81 \%)$ was treated with disease-modifying drugs, without interruption of treatment during the war.

Most patients (82\%) had not experienced clinical relapse in the 12 months before the war, whereas the 
others had between one and four relapses. Seventyseven percent of patients confirmed staying at home for at least 1 week after the war had broken out, and $72 \%$ stayed at home throughout the war period.

\section{Relapses}

The number of confirmed clinical exacerbations during the 33 days of the war and in the 12 previous corresponding month periods (from the 15th of a month to the 14th of the following month) that preceded the war is presented in Figure 1. Eighteen relapses occurred during the war compared with one to six relapses in comparable time periods over the 12 months preceding the war ( $p<0.001-0.02$, McNemar tests). There was no increase in relapse rates during the 3 months that followed the war compared with the same period of the previous year $(p=0.58, \mathrm{McNemar}$ test $)$. All relapses were treated with corticosteroids either at the hospital or at home, ${ }^{15}$ except for three war-related relapses in which steroid treatment has been recommended but patients decided to avoid it.

\section{Perceived Stress}

The proportion of patients with increased perceived stress scores (3 or 4 ) and the percentage of patients reporting high levels of distress (3 or 4) associated with specific war events are presented in Figure 2. Only significant comparisons are shown to simplify the presentation. The percentage of patients reporting the experience of intense subjective stress was greater among patients with wartime relapse compared with remission patients ( 44 vs $20 \% ; p=0.03$ ). The proportion of patients reporting high levels of distress associated with exposure to rocket attacks and with displacement was greater in relapse patients (67 vs $42 \%$, $p=0.05$; and 33 vs $11 \%, p=0.02$, respectively). In addition, a greater percentage of relapse patients reported stress caused by perceived life threat (33 vs $15 \% ; p=0.08)$.

We found no significant difference between the relapse and remission groups in the proportion of patients who reported adverse life events unrelated to the war either throughout the month of the military activity or during the preceding year ( 44 vs $34 \%$, not significant, and 50 vs $39 \%$, not significant, respectively).

Notably, the relapsing and remission groups were similar for age, sex, duration of illness, and Expanded Disability Status Scale score.

\section{Discussion}

Our study suggests that war stress is associated with increased risk for MS exacerbations and provides further support to the alleged relation between psychological stress and MS disease activity, in line with most previous related studies. ${ }^{6,14}$ Notably, previous studies of the impact of psychological stress focused on daily hassles and life difficulties of moderate intensity, ${ }^{4-}$ 7,16,17 whereas this study demonstrates the potential negative impact of exposure to life-threatening situations on the incidence of MS relapses.

It could be argued that relapse patients are expected to report more subjective stress merely because of the profound psychological impact of the exacerbation itself. ${ }^{18}$ We addressed this potential confounder by asking patients how stressful were specific war events, thus making them focus on war-related, rather than diseaserelated, stressors.

Although we found an association between warrelated psychological stress and MS exacerbation, it

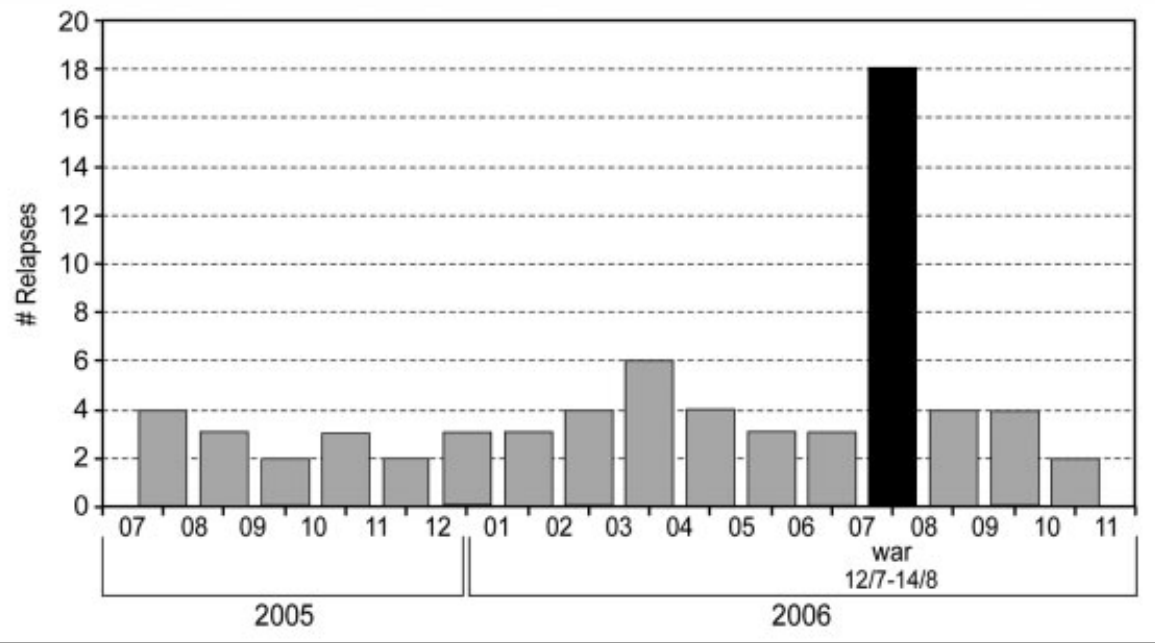

Fig 1. Number of relapses per month. Eighteen relapses occurred during the 33 days of the war compared with one to six relapses in comparable time periods over the 12 months preceding the war. There was no increase in relapse rates during the 3 months that followed the war compared with the same period of the previous year. 


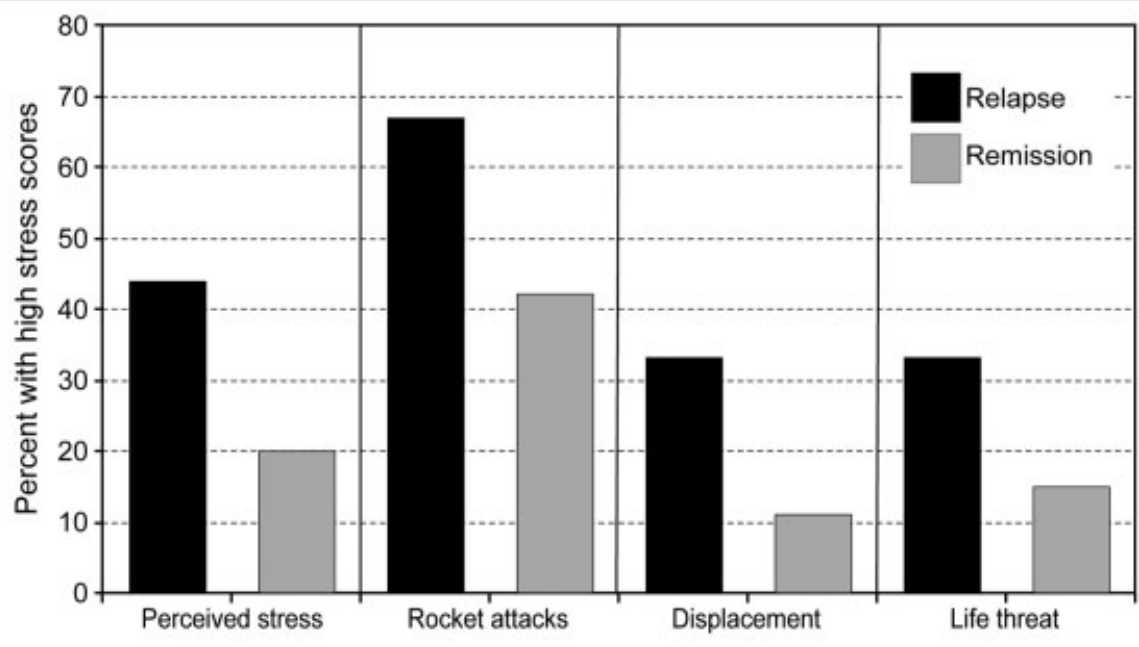

Fig 2. Percentage of patients with high stress scores on the perceived stress scale and regarding specific war events. The percentage of patients reporting the experience of intense subjective stress was greater among patients with wartime relapse (black bars) compared with remission patients (gray bars). The proportion of patients reporting high levels of distress associated with exposure to rocket attacks, displacement, and perceived life threat was also greater in relapse patients.

does not necessarily imply that stress can cause MS attacks. It cannot be ruled out categorically that perceiving war-related external events as stressful is the result, rather than the cause, of MS disease activity, or that both outcomes reflect a common causal factor. Yet, if this were the case, it would be hard to explain the observed increase in the number of severe relapses precisely during the war.

Our observation of the increase in MS-related morbidity during the war is in contrast with a former report of reduction in exacerbations among a group of Israeli MS patients during and after the Persian Gulf War of $1991 .^{8}$ The small number $(\mathrm{n}=32)$ of patients might have biased the results of that study. Furthermore, the authors presume that their patients were under psychological distress without including a measure of subjective stress. They also did not mention how many patients stayed in the assaulted region and whether displacement, if it happened, was stressful. Therefore, a question remain whether part of the patient population of that study did not leave the bombarded district, gaining substantial stress relief.

This study's sample size is considerably larger. Furthermore, after specific inquiry, we determined that $77 \%$ of our participants stayed in the bombed areas for at least 1 week, and $72 \%$ stayed home throughout the war period. It is evident from our results that a subjective war-related sense of distress was heterogeneous among our patients, illustrating a potential bias in presuming that all who have been exposed to war have also been distressed to a similar extent by the complex circumstances of military threat. We also found that experiencing displacement as stressful was more common among relapsing patients, implying that leaving the bombarded area did not necessarily entail stress dissipation for all patients, and that the associated strain might have exacerbated our respondents' state of health.

Previous studies report exacerbations to occur several weeks to several months after stressor onset, and also after the stressful situation was over. ${ }^{4,5,14,16}$ Similarly, it was demonstrated that new gadolinium-enhancing brain lesions occurred 8 weeks after stressor onset in a group of $36 \mathrm{MS}$ patients. ${ }^{3}$ Interestingly, in Mohr and colleagues' study, ${ }^{3}$ the association was with milder stressor events rather than with more severe stressors. The prevailing explanation for this delay is that stressful situations result in aberrant activation of the hypothalamic-pituitary-adrenal axis in a way that frequently results in increased levels of cortisol production. ${ }^{19}$ Thus, as cortisol levels increase after the onset of a stressful situation, there would be increased control over the pathological inflammatory process in MS patients. The stress resolution hypothesis ${ }^{20}$ suggests that the dissipation of the stress with the accompanied decrease in cortisol levels facilitates the development of active inflammation and increases the risk for an exacerbation a few weeks after stress onset.

Notably, 18 of our patients relapsed during the war, and 8 of these patients evacuated their homes before the conflict was ended. One may wonder whether the displacement from home of these eight patients might have conferred some stress relief. However, six of eight denoted high subjective stress regarding their displacement (see Fig 2). Thus, it is unlikely that the stress resolution hypothesis would apply to these circumstances.

Our findings suggest that increased MS relapse rate 
may occur during times when a stressful process is still at its peak; our patients reported MS exacerbations while their places of residence were still battered. A biological model that has been investigated and could potentially explain stress effects on MS symptoms within days involves the acute activation of brain mast cells and increased blood-brain barrier permeability. ${ }^{20-23}$ This would suggest that acute stress might have a permissive effect on MS exacerbations by facilitating blood-brain barrier breakdown through corticotropin-releasing hormone-mediated mast-cell activation and release of vasoactive peptides, such as histamine and mast-cell-specific tryptase. ${ }^{23}$ Such biological mechanism may be relevant to our observation of increased MS relapse rate during the war, at times of maximal stress.

Interestingly, although more than $80 \%$ of the patients were taking disease-modifying agents, this apparently did not prevent the stress-related increase in MS attacks. The predominant mechanisms of action proposed for these immunomodulatory drugs are reduction of T-cell activation, proliferation, and differentiation to Th1 cells. These findings may imply that other mechanisms, beyond T-cell-related aberrant immune reactivities, may be involved in the immunopathogenesis of stress-related exacerbations.

Accumulating evidence indicates that the two major stress-response systems, namely, the hypothalamopituitary-adrenal axis and the autonomic nervous system, are impaired in MS. These appear to be part of the dysregulation of the neuroendocrine-immune networks, suggested to play a role in the pathogenesis and activity of MS. ${ }^{24,25}$ For example, immune cells of MS patients appear to have reduced expression and function of glucocorticoid receptors, entailing permissive effects to inflammation. ${ }^{24-26}$ Also, disturbed intracellular signaling in response to sympathetic stimulation in MS $^{27,28}$ appears to contribute to increase in Th1driven autoimmunity. ${ }^{24}$ Although it is tempting to speculate that malfunction of the stress-response systems may explain the stress-relapse association, it is not yet clear how the increase in stress triggers inflammation, and there is yet much to elucidate regarding the response to stress, depending on the nature, degree, and duration of the stressor, as well as the background and psychological characteristics of the individual.

It has been claimed that exposure to persistent stress is associated with reduced number and function of glucocorticoid receptors on immune cells. ${ }^{29,30}$ Considering that 1 week of daily stress may be sufficient to induce glucocorticoid resistance in peripheral immune cells, ${ }^{29}$ it is possible that this mechanism has played a role in the findings of this study, namely, increased relapse rate at the weeks of actual acute stress. Notably, the proportion of patients who reported stressful life events in the year that preceded the war was similar in the relapse and remission groups, and the Expanded Disability Status Scale score of both groups was not statistically different. Thus, there is no reason to suspect that the relapse patients were under greater chronic prewar stress, either MS related or unrelated.

This study focused on clinical assessment of the impact of war and related stress on disease activity of patients with MS. Future research is warranted to better clarify the possible mechanisms that underlie the alleged increase in disease activity at times of stress. Such studies should be planned to include assessment of biomarkers (including corticosteroids and other indicators of stress response) and magnetic resonance imaging parameters of disease activity.

In conclusion, our data suggest that civilian exposure to war stress and the resulting subjective sense of distress are associated with increased risk for MS exacerbation. The clinical manifestations of MS disease activity may be apparent during times when a stressful process is still at its peak. These findings provide insight to stress-related risk factors associated with relapses of MS and suggest a potential role for applying preventive and tailored interventions, behavioral and pharmacological, in populations at risk among patients with MS.

This work was supported by the Trauma \& Military Medicine Research fund, Technion (200915, A.M.).

We thank I. Lavi for helpful assistance with the data assessment and statistical analysis.

\section{References}

1. Charcot JM. Lectures on diseases of the nervous system. London: New Sydenham Society, 1877.

2. Goodin DS, Ebers GC, Johnson KP, et al. The relationship of MS to physical trauma and psychological stress. Neurology 1999;52:1737-1748.

3. Mohr DC, Goodkin DE, Bacchetti P, et al. Psychological stress and the subsequent appearance of new brain MRI lesions in MS. Neurology 2000;55:55-61.

4. Ackerman KD, Heyman R, Rabin BS, et al. Stressful life events precede exacerbations of multiple sclerosis. Psychosom Med 2002;64:916-920.

5. Buljevac D, Hop WCJ, Reedeker W, et al. Self reported stressful life events and exacerbations in multiple sclerosis: prospective study. BMJ 2003;327:646-650.

6. Mohr DC, Hart SL, Julian L, et al. Association between stressful life events and exacerbation in multiple sclerosis: a metaanalysis. BMJ 2004;328:731-736.

7. Brown RF, Tennant CC, Sharrock M, et al. Relationship between stress and relapse in multiple sclerosis: part I. Important features. Mult Scler 2006;12:453-464.

8. Nisipeanu P, Korczyn AD. Psychological stress as risk factor for exacerbations in multiple sclerosis. Neurology 1993;43: $1311-1312$.

9. The second Lebanon War 2006. Israel Ministry of Foreign Affairs. Available at: www.mfa.gov.il/MFA/History/Modern+ History/Israel+wars. Accessed. 
10. Polman CH, Reingold SC, Edan G, et al. Diagnostic criteria for multiple sclerosis: 2005 revisions to the "McDonald criteria." Ann Neurol 2005;58:840-846.

11. Taillefer MV, Roullet E, Cesaro P, Alperovitch A. Validation of self-reported neurological disability in multiple sclerosis. Int J Epidemiol 1994;23:148-154.

12. McDonald WI, Compston A, Edan G, et al. Recommended diagnostic criteria for multiple sclerosis: Guidelines from the International Panel on the Diagnosis of Multiple Sclerosis. Ann Neurol 2001;50:121-127.

13. Cohen S, Kamarck T, Mermelstein R. A global measure of perceived stress. J Health Soc Behav 1983;24:385-396.

14. Brown RF, Tennant CC, Dunn SM, Pollard JD. A review of stress-relapse interactions in multiple sclerosis: important features and stress-mediating and -moderating variables. Mult Scler 2005;11:477-484.

15. Steinmetz D, Edelstein H, Dishon S, et al. Hospital-at-home as a solution for the treatment requirements of acute exacerbation in multiple sclerosis. Harefuah 2001;140:603-606.

16. Sibley WA. Risk factors in multiple sclerosis. In: Raine CS, McFarland HF, Tourtellotte WW, eds. Multiple sclerosis: clinical and pathogenetic basis. London: Chapman \& Hall, 1997: $141-148$.

17. Grant I, Brown GW, Harris T, et al. Severely threatening events and marked life difficulties preceding onset or exacerbation of multiple sclerosis. J Neurol Neurosurg Psychiatry 1989; 52:8-13.

18. Halper J. The psychosocial effect of multiple sclerosis: the impact of relapses. J Neurol Sci 2007;256(suppl 1):S34-S38.

19. Chrousos GP. The hypothalamic-pituitary-adrenal axis and immune-mediated inflammation. N Engl J Med 1995;332: 1351-1362.

20. Mohr CM, Pelletier D. A temporal framework for understanding the effects of stressful life events in inflammation in patients with multiple sclerosis. Brain Behav Immun 2006;20:27-36.
21. Theoharides TC. Mast cells and stress-a psychoneuroimmunological perspective. J Clin Psychopharmacol 2002;22: 103-108.

22. Zappulla JP, Arock M, Mars LT, Liblau RS. Mast cells: new targets for multiple sclerosis therapy? J Neuroimmunol 2002; 131:5-20.

23. Theoharides TC, Konstantinidou AD. Corticotropin-releasing hormone and the blood-brain-barrier. Front Biosci 2007;2: 1615-1628.

24. Gold SM, Mohr DC, Huitinga I, et al. The role of stressresponse systems for the pathogenesis and progression of MS. Trends Immunol 2005;26:644-652.

25. Heesen C, Mohr DC, Huitinga I, et al. Stress regulation in multiple sclerosis-current issues and concepts. Mult Scler 2006;12:1-6.

26. DeRijk RH, Eskandari F, Sternberg EM. Corticosteroid resistance in a subpopulation of multiple sclerosis patients as measured by ex vivo dexamethasone inhibition of LPS induced IL-6 production. J Neuroimmunol 2004;151:180-188.

27. Giorelli M, Livrea P, Trojano M. Post-receptorial mechanisms underlie functional disregulation of beta2-adrenergic receptors in lymphocytes from multiple sclerosis patients. J Neuroimmunol 2004;155:143-149.

28. Vroon A, Kavelaars A, Limmroth V, et al. G protein-coupled receptor kinase 2 in multiple sclerosis and experimental autoimmune encephalomyelitis. J Immunol 2005;174:4400-4406.

29. Stark JL, Avitsur R, Padgett DA, et al. Social stress induces glucocorticoid resistance in macrophages. Am J Physiol Regul Integr Comp Physiol 2001;280:1799-1805.

30. Miller GE, Cohen S, Ritchey AK. Chronic psychological stress and the regulation of pro-inflammatory cytokines: a glucocorticoid-resistance model. Health Psychol 2002;21: 531-541. 